\title{
BMJ Open Protocol for a multicentre, prospective cohort study of practice patterns and clinical outcomes associated with emergency department sedation for mechanically ventilated patients: the ED-SED Study
}

\author{
Brian M Fuller, ${ }^{1}$ Nicholas M Mohr, ${ }^{2}$ Brian W Roberts, ${ }^{3}$ Christopher R Carpenter, ${ }^{4}$
} Marin H Kollef, ${ }^{5}$ Michael S Avidan ${ }^{6}$

To cite: Fuller BM, Mohr NM, Roberts BW, et al. Protocol for a multicentre, prospective cohort study of practice patterns and clinical outcomes associated with emergency department sedation for mechanically ventilated patients: the ED-SED Study. BMJ Open 2018;8:e023423. doi:10.1136/ bmjopen-2018-023423

- Prepublication history for this paper is available online. To view these files, please visit the journal online (http://dx.doi. org/10.1136/bmjopen-2018023423).

Received 10 April 2018 Revised 1 August 2018 Accepted 13 September 2018

Check for updates

(c) Author(s) (or their employer(s)) 2018. Re-use permitted under CC BY-NC. No commercial re-use. See rights and permissions. Published by BMJ.

For numbered affiliations see end of article.

Correspondence to

Dr Brian M Fuller;

fullerb@wusm.wustl.edu

\section{ABSTRACT}

Introduction In mechanically ventilated patients, sedation strategies are a major determinant of outcome. The emergency department (ED) is the earliest exposure to mechanical ventilation for hundreds of thousands of patients annually in the USA. The one retrospective study that exists regarding ED sedation for mechanically ventilated patients showed a strong association between deep sedation in the $E D$ and worse clinical outcomes. This finding suggests that the ED may be an optimal location to study the impact of early sedation on outcome, yet a lack of prospective studies represents a knowledge gap in this arena. This protocol describes a prospective observational study aimed at further characterising ED sedation practices and assessing the relationship between ED sedation and clinical outcomes. An association between ED sedation and clinical outcomes across multiple sites would suggest the need for changes in the current sedation strategies used in the $\mathrm{ED}$, and provide evidence for future interventional studies in this field.

Methods and analysis This is a multicentre, prospective cohort study testing the hypothesis that deep sedation in the ED is associated with worse clinical outcomes. A cohort of over 300 mechanically ventilated ED patients will be included. The primary outcome is ventilator-free days, and secondary outcomes include hospital mortality, incidence of acute brain dysfunction and lengths of stay. Multivariable linear regression will test the hypothesis that deep sedation in the $E D$ is associated with a decrease in ventilator-free days. Ethics and dissemination Approval of the study by the Institutional Review Board (IRB) at each participating site has been obtained prior to data collection on the first patient. This work will be disseminated by publication of peer-reviewed manuscripts, presentation in abstract form at scientific meetings and data sharing with other investigators through academically established means.

\section{INTRODUCTION}

\section{Background and rationale}

Provision of analgesia and sedation is ubiquitous in the care of mechanically ventilated
Strengths and limitations of this study

- This study is the first to examine prospectively the impact of emergency department (ED) sedation on clinical outcomes.

- The observational design will allow the enrolment of a large sample of diverse patients, which will add significantly to the knowledge base regarding current ED sedation practices.

The multicentre nature of the study will increase external validity of the findings.

- An observational design can only describe associations and not causation.

- The study will rely on real-world assessments and documentation of sedation depth and the presence of delirium, which could be prone to inaccuracies.

patients. While sedation was historically viewed as a means to adapt the patient to the ventilator and facilitate care, recent studies demonstrate that sedation strategies (eg, depth of sedation and medications delivered) are highly influential on clinical outcomes in mechanically ventilated patients. ${ }^{12}$ Given this, guidelines for intensive care unit (ICU) sedation recommend: (1) maintaining light levels of sedation unless clinically contraindicated; (2) objectively monitoring depth of sedation; (3) using non-benzodiazepine sedation strategies and (4) routinely monitoring for the presence of delirium. ${ }^{1}$

The majority of data in this domain comes from randomised controlled trials (RCTs) which enrolled patients after 48-96hours of mechanical ventilation, or observational data centred on an entire ICU stay. ${ }^{3}$ The early period of mechanical ventilation (ie, in the emergency department (ED) and first 
48 hours in ICU) has been largely ignored, yet may be particularly influential on outcome. ${ }^{4}$ In the most comprehensive evaluation of the world's literature to date, our group previously showed a strong association between early (within 48 hours of instituting mechanical ventilation) sedation depth and outcome. ${ }^{3}$ Early light sedation was associated with lower hospital mortality $(9.2 \%)$ versus deep sedation (27.6\%) (OR 0.34, 95\% CI 0.21 to 0.54 ), $\mathrm{p}<0.001$. The frequency of delirium was $28.7 \%$ in lightly sedated patients versus $48.4 \%$ in the deep sedation group $(\mathrm{p}=0.11)$. Early light sedation was also associated with fewer mechanical ventilator days (mean difference, -2.1 (95\% CI -3.6 to -0.5$), \mathrm{p}=0.008$ ) and shorter ICU lengths of stay (mean difference, -3.0 days $(95 \% \mathrm{CI}-5.4$ to -0.6$)$ ), $\mathrm{p}=0.02{ }^{3}$ This systematic review and meta-analysis also identified a critical knowledge gap regarding early sedation and its potential impact on outcome, as the majority of data was from retrospective cohort studies, and only two pilot RCTs ( $\mathrm{n}=97$ patients) have been conducted. ${ }^{56}$

The ED is the most proximal time period of mechanical ventilation for many critically ill patients, yet little attention has been given to the impact that ED-based sedation may have on outcome. Our preliminary data suggest that the ED may be an optimal location to study the impact of early sedation for several reasons. ${ }^{7}$ First, deep sedation in the ED is common (64\%), with a median Richmond Agitation-Sedation Scale (RASS) of -3.0 ( -4.0 to -2.0$)$. Evidence shows that this early oversedation can extend for days in the ICU (sedation overshoot). ${ }^{8}$ This fact, paired with our data, suggests that an ED-based goal-oriented sedation intervention could not only reduce the negative effects of deep sedation in the ED, but reduce sedation overshoot early in the ICU as well. Second, deep sedation in the ED is associated with higher mortality, and more ventilator and ICU days. ${ }^{7}$ Third, approximately two-thirds of mechanically ventilated ED patients receive benzodiazepines, a strategy shown to increase the incidence of delirium, as well as the duration of mechanical ventilation and ICU length of stay. Targeting early sedation in the ED seems needed, and may be an effective strategy to reduce complications in this vulnerable cohort. This has not been a topic of previous research.

In addition to the clinical outcome data mentioned above, there is strong biological rationale for the avoidance of deep sedation by targeting the ED. Not only does sedation exert profound effects on the brain, preclinical data demonstrate that sedation has negative pleiotropic effects on other organ systems. In the gastrointestinal tract, sedatives and opiates can imbalance the microbiome, compromise intestinal barrier function, induce bacterial translocation and increasing microbial virulence. ${ }^{9}$ In the microcirculation, deep sedation induces deterioration in vasomotor function and response to ischaemia, suggesting sedation strategy could affect tissue perfusion at the microcirculatory level. ${ }^{10}$ In the immune system, sedatives can inhibit neutrophil and macrophage function, decrease bacterial clearance and increase mortality in infected animals. ${ }^{11-17}$ Significant to the paradigm of early deep sedation, these immunomodulatory effects can be seen early, within 1-2 hours. ${ }^{18}$

The data regarding ED sedation only exist from our centre. It is unknown if our results are generalisable to other EDs, and this represents a significant knowledge gap. Therefore, prior to the planning of any potential interventional studies, we feel it important to further investigate the current state of ED sedation in critically ill mechanically ventilated patients. Given the clinical outcome data associated with early sedation, as well as the preliminary ED-based data from our centre, we designed this study with the objectives to: (1) further characterise modern ED sedation practices across multiple sites and (2) assess the relationship between ED sedation and clinical outcomes.

\section{Specific aims}

\section{Aim 1}

To assess the relationship between ED sedation (depth and agents used) and clinical outcomes. We hypothesise that deep sedation in the ED will be independently associated with significant differences in clinical outcomes after admission to the ICU.

\section{Aim 2}

To characterise ED sedation practices for mechanically ventilated patients across a diverse cohort of academic institutions.

\section{Significance}

It is likely that sedation in mechanically ventilated patients is highly influential on patient-centred outcomes, such as ventilator-free days, mortality, delirium incidence and lengths of stay. Accurate assessment of ED sedation and improved understanding of outcomes associated with ED sedation can help guide decision making, improve patient care and assist in the planning of future clinical studies. It appears to be common for patients to be deeply sedated in the ED, which is discordant with ICU-based guideline recommendations. ${ }^{17}$ However, it is possible that sedation requirements and approaches may be distinctly different in the ED from those in the ICU, and existing guidelines may not be applicable. Our initial data show that ED-based sedation could be highly influential on outcome. Going forward, a strong association between ED sedation and clinical outcomes across multiple sites would suggest the need for changes in the current standard of care in the $\mathrm{ED}$, and provide evidential need for intervention studies in this area.

\section{METHODS AND ANALYSIS}

\section{Study design}

This is a multicentre, prospective cohort study. Using a cross-sectional approach, data for each centre will be collected during a 1-month time period between 1 June and 31 August 2018. A schematic of the design appears in figure 1 . 


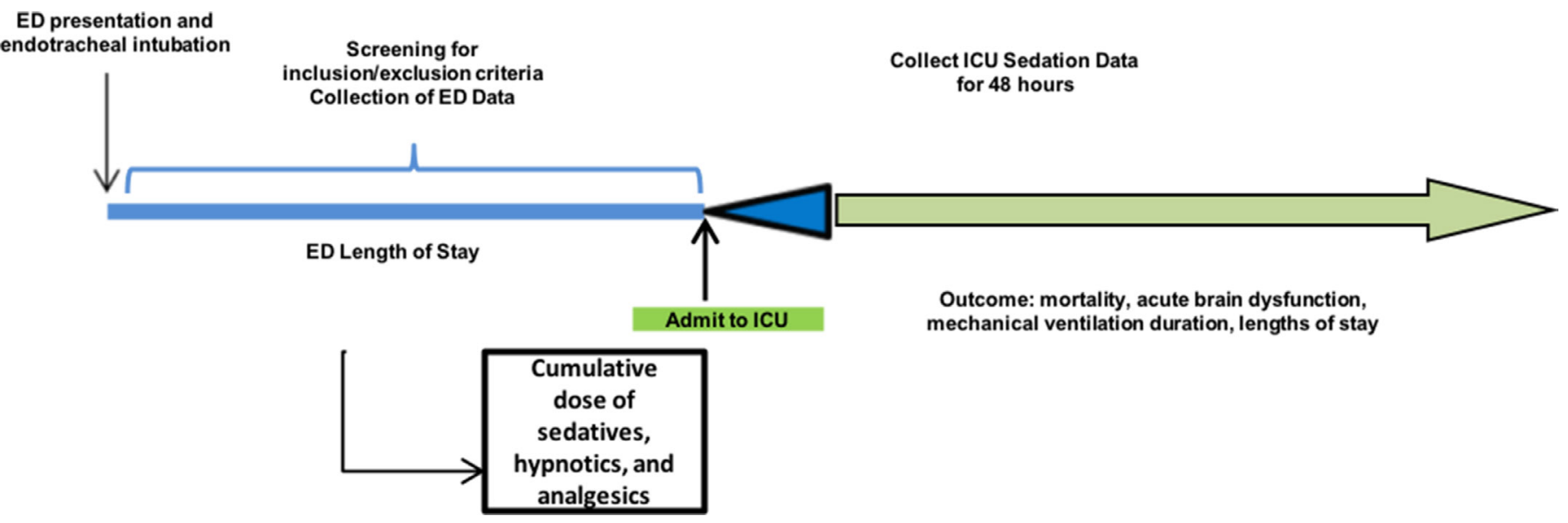

Figure 1 Schematic of study design. ED, emergency department; ICU, intensive care unit.

\section{Study population}

The target population for this study is mechanically ventilated patients in the ED. Inclusion criteria are: (1) receipt of invasive mechanical ventilation in the ED. Exclusion criteria are: (1) death or discontinuation of mechanical ventilation within 24 hours of presentation; (2) transfer to another hospital from the ED; (3) presence of neurological injury as the indication for mechanical ventilation (eg, acute cerebrovascular accident, traumatic brain injury, status epilepticus or sudden cardiac arrest) and (4) chronic/home mechanical ventilation. Presence of neurological injury is an exclusion criterion as patients with neurological injury can have depressed levels of consciousness that is independent of sedation, and therefore serve as a confounder between sedation depth and clinical outcomes. Patients on chronic/home ventilation may require vastly different sedation approaches (ie, no sedation at all), given their chronic condition. Also, the ability to calculate ventilator-free days in these patients may not be possible.

Patients will be recruited exclusively from the ED, and enrolled without regard to gender or race. Based on the multicentre nature of this study, and the demographics of the patient population routinely presenting to the ED, we expect a diverse patient population. We, therefore, expect that the study findings will hold external validity and be applicable to the community as a whole.

\section{Patient and public involvement}

The patients in this study were not involved in the development of the research question or study design, and will not be involved in recruitment or conduct of the study.

\section{Data}

We anticipate collecting the following baseline characteristics: age, gender, race, weight, height, pre-existing comorbid conditions, vital signs at presentation and pertinent laboratory variables. Illness severity will be assessed with the modified Sequential Organ Failure Assessment (SOFA) score. This omits the Glasgow Coma Scale (GCS) component of the SOFA score, which has poor interrater agreement; it is also collinear to measurements of sedation depth. ${ }^{19-21}$ Comorbid conditions will include: dementia, diabetes mellitus, cirrhosis, congestive heart failure, end-stage renal disease, chronic obstructive pulmonary disease, immunosuppression, malignancy, alcohol abuse and history of psychiatric illness (eg, schizophrenia, bipolar, major depression or anxiety).

ED process of care variables will include: ED length of stay, blood product transfusion, antibiotics, central venous catheter placement and vasopressor infusion. Data related to mechanical ventilation will include: location of intubation (ie, prehospital or ED), indication for mechanical ventilation, ventilator mode, tidal volume, positive end-expiratory pressure, set respiratory rate, fraction of inspired oxygen, peripheral oxygen saturation, peak airway pressure and inspiratory plateau pressure.

Data related to sedation in the ED will include: neuromuscular blockers and induction agents administered to facilitate endotracheal intubation. Subsequent medications related to analgesia and sedation in the ED will also be collected, and will include: opiates, benzodiazepines, propofol, ketamine, dexmedetomidine, etomidate, haloperidol, quetiapine and neuromuscular blocking agents. Medications administered for the management of analgosedation during the first 48 hours of ICU admission will also be collected. All antipsychotic agents, such as quetiapine and haloperidol, will be collected.

Sedation depth in the ED will be recorded. It is recognised that multiple sedation scales exist for monitoring sedation depth in mechanically ventilated patients. Given the observational and pragmatic design of the study, sedation depth will be monitored according to the standard operating procedures already in place at an existing site. This may include monitoring of sedation depth with scales such as the (RASS; deep sedation defined as a score of -3 to -5 ; preferential scale) or the Riker Sedation-Agitation Scale (deep sedation defined as a score of 2 or 1) ${ }^{22}$ If more than one sedation depth per patient is documented in the ED, then the median value will be used. ${ }^{7}$

As sedation in the ED for mechanically ventilated patients has not been a research or clinical focus until recently, it is also recognised that some EDs may not have 


\begin{tabular}{|c|c|c|c|c|c|}
\hline & $\begin{array}{l}\text { ED } \\
\text { presentation } \\
\text { and } \\
\text { initiation of } \\
\text { mechanical } \\
\text { ventilation }\end{array}$ & $\begin{array}{l}\text { Admit } \\
\text { to ICU }\end{array}$ & $\begin{array}{l}\text { ICU } \\
\text { day } \\
1\end{array}$ & $\begin{array}{l}\text { ICU } \\
\text { day } \\
2\end{array}$ & $\begin{array}{l}\text { Day } \\
28\end{array}$ \\
\hline $\begin{array}{l}\text { Inclusion/exclusion } \\
\text { criteria }\end{array}$ & $x$ & & & & \\
\hline Demographics & $\mathrm{X}$ & & & & \\
\hline Comorbidities & $x$ & & & & \\
\hline $\begin{array}{l}\text { Illness severity } \\
\text { scores }\end{array}$ & $x$ & & & & \\
\hline $\begin{array}{l}\text { Vitals and } \\
\text { laboratory data }\end{array}$ & $x$ & & & & \\
\hline $\begin{array}{l}\text { ED treatment } \\
\text { variables }\end{array}$ & $\mathrm{X}$ & & & & \\
\hline ED ventilator data & $\mathrm{X}$ & & & & \\
\hline ED sedation data & $\mathrm{X}$ & & & & \\
\hline Depth of sedation* & $x$ & & $x$ & $x$ & \\
\hline ICU sedation data & & & $x$ & $x$ & \\
\hline CAM-ICU & & & $\mathrm{X}$ & $\mathrm{X}$ & \\
\hline $\begin{array}{l}\text { Acute brain } \\
\text { dysfunction }\end{array}$ & & & $x$ & $x$ & \\
\hline Ventilator-free days & & & & & $x$ \\
\hline $\begin{array}{l}\text { Other secondary } \\
\text { outcomes }\end{array}$ & & & & & $\mathrm{X}$ \\
\hline
\end{tabular}

*Preferentially assessed with RASS Score; SAS or GCS may also be used, per local institutional procedures.

CAM, confusion assessment method; ED, emergency department; GCS, Glasgow Coma Scale; ICU, intensive care unit; RASS, Richmond Agitation-Sedation Scale; SAS, Riker Sedation-Agitation Scale.

protocols in place to routinely monitor sedation depth. In this situation, we will use surrogates for depth of sedation, which include a documented GCS (GCS $<9$ defined as deep sedation) ${ }^{23}$ In patients for whom no sedation depth is documented in the ED, the first ICU RASS will be used as a surrogate for the ED depth of sedation, provided that it is measured within the first 3 hours of ICU admission, congruent with our prior approach. ${ }^{7}$ Use of an early ICU RASS score as a reliable surrogate for ED RASS is supported by previous data demonstrating that sedation depth remains relatively static during the first 24 hours of ICU admission. ${ }^{4}$

Pertinent clinical data after admission, including the first 48 hours of ICU sedation depth, will also be included. Table 1 shows a full description of events for this study.

\section{Outcomes}

Patients will be followed until hospital discharge or death. The primary outcome of interest is ventilator-free days. Secondary outcomes include all cause in-hospital mortality, the presence of acute brain dysfunction during the first 48 hours in the ICU, as well as ICU-free and hospital-free days.

Acute brain dysfunction is a composite outcome composed of delirium and coma. Delirium will be assessed by the Confusion Assessment Method for the ICU (CAM-ICU) per local institutional protocols. This is a highly reproducible and well-validated method for diagnosing delirium in mechanically ventilated patients. Coma will be defined as having all documented RASS (or equivalent sedation scale) scores of -4 (responsive to only physical stimulus) or -5 (unresponsive) during the first 48 hours. We elect to use this composite outcome since both delirium and coma are major categories of cognitive dysfunction. As delirium cannot be assessed during periods of coma, using this composite outcome provides a more accurate event rate for the incidence of acute organ dysfunction of the brain. This secondary outcome will be assessed at 48 hours for two reasons: (1) early sedation within this time frame has been strongly associated with clinical outcome in the ICU ${ }^{42} 25$ and (2) initial sedation approach often extends into this time period, providing a temporal link to the sedation provided in the ED. ${ }^{78}$

Clinical outcomes will be assessed as a function of ED sedation depth, with the a priori hypothesis being that deep sedation will be associated with fewer ventilator-free days, higher mortality, a greater incidence of acute brain dysfunction and longer lengths of stay.

Descriptive outcomes include the sedative and analgesic medications administered in the ED. By assessing this descriptive outcome, we will further characterise ED-based sedation and assess potential targets for future investigation. We will also describe early ICU sedation practices during the first 48 hours after admission. If early ICU sedation appears to be influenced by the initial sedation approach in the $\mathrm{ED}$, this provides further rationale that the ED should be the starting point and target for future studies.

\section{Proposed statistical methods}

Patient characteristics will be assessed with descriptive statistics and frequency distributions. Categorical characteristics will be compared using the $\chi^{2}$ test or Fisher's exact test. Continuous characteristics will be compared using the independent samples t-test or Wilcoxon's rank-sum test.

The primary analysis will examine ventilator-free days as a function of ED sedation depth. A multivariable linear regression model will be constructed to adjust for potentially confounding variables using backward elimination. A priori baseline characteristics with known prognostic significance for mortality in ED mechanically ventilated patients will be included in the model. Other clinically relevant and biologically plausible variable that are statistically significant in univariate analysis at a $p<0.10$ level will also be included in the model. Collinearity will be assessed and the model will use variables that are statistically independent of other variables. All tests will be two tailed, and a $\mathrm{p}<0.05$ will be considered statistically 
significant. Time (in days) to mortality will be assessed with the Kaplan-Meier survival estimate and log-rank test, comparing the deep sedation and light sedation groups.

We will conduct a priori subgroup analyses to further understand the treatment effect and identify subgroups in which heterogeneous treatment effects exist. These subgroups will be based on (but are not limited to): illness severity, indication for mechanical ventilation and specific medications received in the ED or ICU (eg, benzodiazepines, dexmedetomidine). On study completion, additional analyses may be performed post hoc if they are believed to contribute valuable or novel information, or if unforeseen imbalances in the data are present.

\section{Sample size}

We estimate our sample size calculation based on prior work from the ICU which reported the impact of sedation on ventilator-free days, ${ }^{26-29}$ a cohort study regarding early sedation depth and its impact on outcome, ${ }^{4}$ and our single-centre cohort study examining the impact of ED sedation depth on clinical outcome. ${ }^{7}$ We conservatively estimated a difference in mean (SD) ventilator-free days of 2.5 between the two groups: deep sedation (18.5 (8.0)) vs light sedation (21.0 (8.0)). For $80 \%$ power and a $\alpha$ of 0.05 , we will need a total sample size 324 patients (162 per group). Based on our prior work involving mechanically ventilated patients in the $\mathrm{ED}$, we expect approximately $0.75-1.0$ patients to satisfy inclusion and exclusion criteria per site, each day. ${ }^{30} 31$ With 16 centres agreeing to participate, we expect a total sample size that is capable of achieving the objectives of this investigation.

\section{Anticipated results}

We anticipate that deep sedation in the ED will be common, as will the use of benzodiazepines for postintubation sedation. We also anticipate that the presence of deep sedation in the ED will be associated with a reduction in ventilator-free days, higher mortality and a greater incidence of acute brain dysfunction and longer lengths of stay.

\section{Data storage and management}

All data will be entered by site principal investigators (PI) or research assistants and data accuracy will be verified by the study PI. Data quality control measures will include queries to identify missing data, outliers and discrepancies. Only research assistants and site PIs will have access to protected health information. After enrolment, a unique identifier will be assigned to each study subject. The data from all sites will be uploaded and stored using Research Electronic Data Capture, a web-based data management application. All computers will be password protected and encrypted per university policy. The PI will ensure that the anonymity is maintained. Patients will not be identified by name in any reports on this study. The study PI will have access to the final study dataset.

\section{ETHICS AND DISSEMINATION}

\section{Dissemination and data sharing}

To enhance reporting transparency, this study will be reported in accordance with the Strengthening the Reporting of Observational Studies in Epidemiology Statement: Guidelines for Reporting Observational Studies. ${ }^{32}$

Data and resources will be shared with other eligible investigators through academically established means. The datasets used and/or analysed during the study will be available from the corresponding author on reasonable request. Collaboration with others investigators interested in optimising outcomes for mechanically ventilated patients in the ED will be welcomed. The results from this work will be published as a full-length, peer-reviewed manuscript and presented at national meetings.

\section{Strengths and limitations \\ Strengths}

Currently, only one retrospective cohort study has been published regarding the impact of ED sedation on clinical outcome. ${ }^{7}$ The current investigation will add a significant amount of data to this research domain. Further, it will address some of the prior weaknesses in this field: (1) the multicentre nature of the study will enhance external validity of the findings and (2) it will prospectively enrol patients and capture data. This will allow us to track sedation depth as a potential function of illness severity or neurological status with more accuracy, reducing potential confounders related to sedation depth.

\section{Limitations}

This study will have several limitations. As an observational study, it will only be able to demonstrate associations and not causation. We will attempt to enhance causal inference in several ways. We will apply a rigorous multivariable analysis to address potential confounders. A scientific gap will be addressed by enrolling across multiple sites and we will check the consistency of our results with prior work in this field. Finally, we will report all results transparently in accordance with guideline recommendations, which have been shown to improve reporting in observational studies. ${ }^{32}$ The observational cohort study design is also prone to confounders, though this should be reduced somewhat by prospective data collection. We also will have to rely on real-world assessments and documentation of sedation depth and the presence of delirium (CAM-ICU). While these assessments are part of standard ED and ICU protocols for mechanically ventilated patients, without dedicated study team members performing these assessments, there is potential for inaccuracies. However, this does reflect daily practice and may enhance external validity of our findings. The collection of clinical data beyond 48 hours would be advantageous for the association between ED sedation depth and other clinical outcomes, such as organ failure and ICU-acquired infections. Finally, the intent of this investigation is not to produce definitive answers related to ED-based sedation and its impact on outcome, but rather to provide further exploratory data in this area and more preliminary data for 
larger trials. In that regard, this multicentre study could be pivotal in changing how sedation is employed in the ED.

\section{Author affiliations}

${ }^{1}$ Department of Anesthesiology, Division of Critical Care, Division of Emergency Medicine, Washington University School of Medicine in St. Louis, St. Louis, Missouri, USA

${ }^{2}$ Departments of Emergency Medicine and Anesthesiology, Division of Critical Care, Roy J. and Lucille A. Carver College of Medicine, University of lowa, lowa City, lowa, USA

${ }^{3}$ Department of Emergency Medicine, Cooper University Hospital, Camden, New Jersey, USA

${ }^{4}$ Department of Emergency Medicine, Washington University in St. Louis School of Medicine, St. Louis, Missouri, USA

${ }^{5}$ Department of Medicine, Division of Pulmonary and Critical Care Medicine, Washington University in St. Louis School of Medicine, St. Louis, Missouri, USA ${ }^{6}$ Department of Anesthesiology, Washington University School of Medicine in St. Louis, St. Louis, Missouri, USA

Contributors BMF: conception and study design, acquisition of data, analysis and interpretation of data, drafting and revising the manuscript. NMM: study design, acquisition of data, analysis and interpretation of data, drafting and revising the manuscript. BWR: study design, acquisition of data, analysis and interpretation of data, drafting and revising the manuscript. CRC: study design, analysis and interpretation of data, drafting and revising the manuscript. MHK: study design, analysis and interpretation of data, drafting and revising the manuscript. MSA: study design, analysis and interpretation of data, drafting and revising the manuscript. All authors have read and given final approval of the submitted manuscript.

Funding The authors have not declared a specific grant for this research from any funding agency in the public, commercial or not-for-profit sectors.

Competing interests None declared.

Patient consent Not required.

Ethics approval The study protocol has received ethical approval by the Human Research Protection Office/Institutional Review Board at each participating site prior to beginning the study.

Provenance and peer review Not commissioned; externally peer reviewed.

Open access This is an open access article distributed in accordance with the Creative Commons Attribution Non Commercial (CC BY-NC 4.0) license, which permits others to distribute, remix, adapt, build upon this work non-commercially, and license their derivative works on different terms, provided the original work is properly cited, appropriate credit is given, any changes made indicated, and the use is non-commercial. See: http://creativecommons.org/licenses/by-nc/4.0/.

\section{REFERENCES}

1. Barr J, Fraser GL, Puntillo K, et al. Clinical practice quidelines for the management of pain, agitation, and delirium in adult patients in the intensive care unit. Crit Care Med 2013;41:263-306.

2. Jackson DL, Proudfoot CW, Cann KF, et al. A systematic review of the impact of sedation practice in the ICU on resource use, costs and patient safety. Crit Care 2010;14.R59.

3. Stephens RJ, Dettmer MR, Roberts BW, et al. Practice patterns and outcomes associated with early sedation depth in mechanically ventilated patients: A systematic review and meta-analysis. Crit Care Med 2018:46:471-9.

4. Shehabi Y, Bellomo R, Reade MC, et al. Early intensive care sedation predicts long-term mortality in ventilated critically ill patients. Am J Respir Crit Care Med 2012;186:724-31.

5. Shehabi Y, Bellomo R, Reade MC, et al. Early goal-directed sedation versus standard sedation in mechanically ventilated critically ill patients: a pilot study*. Crit Care Med 2013;41:1983-91.

6. Shehabi Y, Chan L, Ismail WN, et al. 869: Early Goal Directed Sedation with Dexmedetomidine vs Standard Sedatives, Randomized Control Trial. Crit Care Med 2013;41:A217.

7. Stephens RJ, Ablordeppey E, Drewry AM, et al. Analgosedation practices and the impact of sedation depth on clinical outcomes among patients requiring mechanical ventilation in the ED: A cohort study. Chest 2017;152:963-71.
8. Jackson DL, Proudfoot CW, Cann KF, et al. The incidence of sub-optimal sedation in the ICU: a systematic review. Crit Care 2009;13:R204.

9. Dickson RP. The microbiome and critical illness. Lancet Respir Med 2016;4:59-72.

10. Lamblin V, Favory R, Boulo M, et al. Microcirculatory alterations induced by sedation in intensive care patients. Effects of midazolam alone and in association with sufentanil. Crit Care 2006;10:R176.

11. Heller A, Heller S, Blecken S, et al. Effects of intravenous anesthetics on bacterial elimination in human blood in vitro. Acta Anaesthesiol Scand 1998;42:518-26.

12. Kelbel I, Koch T, Weber A, et al. Alterations of bacterial clearance induced by propofol. Acta Anaesthesiol Scand 1999;43:71-6.

13. Krumholz W, Endrass J, Hempelmann G. Propofol inhibits phagocytosis and killing of Staphylococcus aureus and Escherichia coli by polymorphonuclear leukocytes in vitro. Can J Anaesth 1994;41:446-9.

14. Laschi A, Descotes J, Tachon P, et al. Adverse influence of diazepam upon resistance to Klebsiella pneumoniae infection in mice. Toxicol Lett 1983;16(3-4):281-4.

15. Massoco C, Palermo-Neto J. Effects of midazolam on equine innate immune response: a flow cytometric study. Vet Immunol Immunopathol 2003;95(1-2):11-19.

16. Smith MA, Hibino M, Falcione BA, et al. Immunosuppressive aspects of analgesics and sedatives used in mechanically ventilated patients: an underappreciated risk factor for the development of ventilatorassociated pneumonia in critically ill patients. Ann Pharmacother 2014; $48: 77-85$

17. Weinert CR, Kethireddy S, Roy S. Opioids and infections in the intensive care unit should clinicians and patients be concerned? $J$ Neuroimmune Pharmacol 2008;3:218-29.

18. Taniguchi $\mathrm{T}$, Yamamoto $\mathrm{K}$, Ohmoto $\mathrm{N}$, et al. Effects of propofol on hemodynamic and inflammatory responses to endotoxemia in rats. Crit Care Med 2000;28:1101-6.

19. Vincent JL, Angus DC, Artigas A, et al. Effects of drotrecogin alfa (activated) on organ dysfunction in the PROWESS trial. Crit Care Med 2003;31:834-40.

20. Vincent J-L, De Mendonça A, Cantraine F, et al. Use of the SOFA score to assess the incidence of organ dysfunction/failure in intensive care units: results of a multicenter, prospective study. Critical care medicine 1998;26:1793-800.

21. Vincent J-L, Moreno R, Takala J, et al. The SOFA (Sepsis-related Organ Failure Assessment) score to describe organ dysfunction/ failure: Springer, 1996.

22. Reade MC, Finfer S. Sedation and delirium in the intensive care unit. N Engl J Med 2014;370:444-54.

23. Tanaka LM, Azevedo LC, Park M, et al. Early sedation and clinical outcomes of mechanically ventilated patients: a prospective multicenter cohort study. Crit Care 2014;18.R156.

24. Shehabi Y, Chan L, Kadiman S, et al. Sedation depth and longterm mortality in mechanically ventilated critically ill adults: a prospective longitudinal multicentre cohort study. Intensive Care Med 2013;39:910-8.

25. Shehabi Y, Bellomo R, Kadiman S, et al. Sedation intensity in the first 48 hours of mechanical ventilation and 180 -day mortality: A multinational prospective longitudinal cohort study. Crit Care Med 2018;46:850-9.

26. Girard TD, Kress JP, Fuchs BD, et al. Efficacy and safety of a paired sedation and ventilator weaning protocol for mechanically ventilated patients in intensive care (Awakening and Breathing Controlled trial): a randomised controlled trial. Lancet 2008:371:126-34.

27. Pandharipande PP, Pun BT, Herr DL, et al. Effect of sedation with dexmedetomidine vs lorazepam on acute brain dysfunction in mechanically ventilated patients: the MENDS randomized controlled trial. JAMA 2007;298:2644-53.

28. Strøm T, Martinussen T, Toft P. A protocol of no sedation for critically ill patients receiving mechanical ventilation: a randomised trial. Lancet 2010;375:475-.

29. Carson SS, Kress JP, Rodgers JE, et al. A randomized trial of intermittent lorazepam versus propofol with daily interruption in mechanically ventilated patients. Crit Care Med 2006;34:1326-32.

30. Fuller BM, Ferguson IT, Mohr NM, et al. Lung-protective ventilation initiated in the emergency department (LOV-ED): A quasiexperimental, before-after trial. Ann Emerg Med 2017;70:406-18.

31. Fuller BM, Mohr NM, Miller CN, et al. Mechanical Ventilation and ARDS in the ED. Chest 2015;148:365-74.

32. von Elm E, Altman DG, Egger M, et al. The strengthening the reporting of observational studies in epidemiology (strobe) statement: Guidelines for reporting observational studies. Ann Intern Med 2007;147:573-7. 